\title{
Lessons learned from the prototypes of the MQXFA Low-Beta Quadrupoles for HL-LHC and status of production in the US
}

\author{
G. Ambrosio, K. Amm, M. Anerella, G. Apollinari, M. Baldini, J. Blowers, R. Bossert, R. Carcagno, D. W. Cheng, \\ G. Chlachidze, L. D. Cooley, J. DiMarco, S. Feher, P. Ferracin, S. Izquierdo Bermudez, P. Joshi, S. Krave, J. Levi- \\ tan, V. Lombardo, J. Lu, M. Marchevsky, V. Marinozzi, J. Muratore, F. Nobrega, T. Page, H. Pan, M. Parker, I. \\ Pong, S. Prestemon, E. Ravaioli, G. Sabbi, J. Schmalzle, H. Song, S. Stoynev, T. Strauss, E. Takala, E. Todesco, D. \\ Turrioni, G. Vallone, P. Wanderer, X. Wang, M.Yu
}

\begin{abstract}
With the successful test of the first two pre-series magnets the US HL-LHC Accelerator Upgrade Project has started production of the MQXFA magnets to be used in Q1/Q3 inner triplet elements of the HL-LHC. This good start comes after the test of two prototypes with limited performance, and it demonstrates the importance of learning from past issues.

Therefore, in this paper we want to share the most important lessons learned so far, focusing on those which may be more interesting for similar projects. We will also present the status of MQXFA fabrication in the US.
\end{abstract}

Index Terms-Accelerator magnets, HL-LHC, Lessons Learned, $\mathrm{Nb}_{3} \mathrm{Sn}$.

\section{INTRODUCTION}

$\mathbf{T}$ he US has started contributing to the high luminosity upgrade of the Large Hadron Collider (HL-LHC) [1] at CERN with the fabrication of the first MQXFA magnets [2] to be used in Q1 and Q3 Inner Triplet elements, through the HL-LHC Accelerator Upgrade Project (AUP) [3].

CERN is planning to make the $\mathrm{Q} 2 \mathrm{a} / \mathrm{b}$ inner triplet elements, which will contain the MQXFB quadrupoles [4]. MQXFA and MQXFB magnets have identical cross-section, although their length is 4.2 and $7.15 \mathrm{~m}$ respectively. The first iteration of the MQXF design was completed by the US LHC Accelerator Research Program (LARP) [5] in collaboration with CERN, and lead to the successful fabrication and test of the first short model (MQXFS1) [6]. After this phase LARP fabricated and assembled two prototypes (MQXFAP1 and MQXFAP2). Subsequently, in collaboration with CERN, AUP finalized the Design Criteria, the MQXF design, the quality control (QC)
TABLE I

MQXF MAIN PARAMETERS

\begin{tabular}{ccccc}
\hline \hline Parameter & Unit & Nominal $^{\mathrm{a}}$ & Ultimate $^{\mathrm{b}}$ & SSL $^{\mathrm{c}}$ \\
\hline Current & $\mathrm{kA}$ & 16.23 & 17.5 & 21.24 \\
I/ISL & $\%$ & 76 & 82 & 100 \\
Gradient & $\mathrm{T} / \mathrm{m}$ & 132.2 & 142.1 & 168.1 \\
Coil peak field & $\mathrm{T}$ & 11.3 & 12.1 & 14.5 \\
Stored energy & $\mathrm{MJ} / \mathrm{m}$ & 1.15 & 1.32 & 1.89 \\
Curr. sharing temp. & $\mathrm{K}$ & 7.0 & 6.0 & 1.9 \\
Diff. Inductance & $\mathrm{mH} / \mathrm{m}$ & 8.26 & 8.23 & 8.13 \\
SC. current density & $\mathrm{A} / \mathrm{mm}^{2}$ & 1573 & 1695 & 2059 \\
Eng. current density & $\mathrm{A} / \mathrm{mm}^{2}$ & 715 & 771 & 936 \\
\hline
\end{tabular}

Main MQXF parameters for operation at $1.9 \mathrm{~K}$

${ }^{a}$ Nominal current is for $\mathrm{LHC}$ operation at $7 \mathrm{TeV}$ per beam

bUltimate current is for LHC operation at $7.5 \mathrm{TeV}$ per beam

${ }^{\mathrm{c}}$ Short Sample Limit (SSL) is for minimal conductor requirement

${ }^{\mathrm{d} D i f f e r e n t i a l ~ i n d u c t a n c e ~ a t ~ h i g h ~ f i e l d ~}$

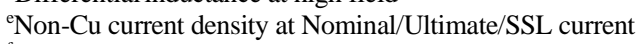

${ }^{\mathrm{f}}$ Current density in whole conductor (non- $\mathrm{Cu}$ and $\mathrm{Cu}$ )

procedures, and started pre-series magnet fabrication. Table I presents the main MQXF parameters.

The cold tests of MQXFA prototypes gave mixed results. Nonetheless they provided very useful feedback for finalizing design, assembly and QC procedures. The first pre-series magnets (MQXFA03 and MQXFA04) incorporated all lessons learned from the prototypes and met all key requirements during test.

In this paper we present the main issues found during prototyping phase, corrective and preventive actions, and lessons learned.

D. W. Cheng, P. Ferracin, M. Marchevsky, H. Pan, I. Pong, S. Prestemon, G. Sabbi, G. Vallone, X. Wang, are with Lawrence Berkeley National Laboratory, Berkeley, CA 94720 USA.

L. D. Cooley, J. Levitan, J. Lu are with the Applied Superconductivity Center, National High Magnetic Laboratory, Tallahassee, FL 32310, USA and with FSU.

S. Izquierdo Bermudez, E. Ravaioli, E. T. Takala, E. Todesco are with CERN, Geneve, Switzerland.

Digital Object Identifier will be inserted here upon acceptance.
This work was supported by the U.S. Department of Energy, Office of Science, (AUP) and by the High Luminosity LHC project at CERN.

G. Ambrosio, G. Apollinari, M. Baldini, J. Blowers, R. Bossert, R. Carcagno, , G. Chlachidze, J. DiMarco, S. Feher, S. Krave, V. Lombardo, V. Marinozzi, F. Nobrega, T. Page, M. Parker, S. Stoynev, T. Strauss, D. Turrioni, M.Yu, are with Fermi National Accelerator Laboratory, Batavia, IL 60510 USA, (e-mail: giorgioa@fnal.gov).

K. Amm, M. Anerella, P. Joshi, J. Muratore, J. Schmalzle, H. Song, P. Wanderer, are with Brookhaven National Laboratory, Upton, NY 11973 USA. 


\section{LESSONS LEARNED ABOUT ELECTRICAL QC TESTS}

The MQXF cable insulation consists of a $145 \mu \mathrm{m}$ thick S2glass sleeve braided on the cable. After heat treatment the coil is vacuum impregnated with epoxy. Before epoxy impregnation, quench heaters with 50 um thick polyimide insulation [7] and S2-glass cloths are set on coil outer surface.

The test of the first MQXFA prototype (MQXFAP1) [8], performed at BNL Vertical Test Facility, was stopped after detection of a coil-to-ground short when magnet training had almost reached $17.5 \mathrm{kA}$. Test data analysis and autopsy [7] showed that this short had been caused by a series of issues, which we summarize in chronological order: 1) a coil (QXFP5) used in this magnet had an experimental S2-glass cloth (provided by a new vendor and with a denser fabric than all cloths previously used) on its outer surface that resulted in a non-conforming epoxy impregnation due to dry spots; 2) during the first quench, the test facility burst valve ruptured forcing a thermal cycle; 3 ) at room temperature heater-to-coil high-voltage withstand (Hipot) tests were performed at the pre-cooldown values $(2.5 \mathrm{kV})$ and one heater of coil QXFP5 failed; 4) Hipot tests were repeated on the failed heater to assess its reduced insulation strength but ended up causing further degradation; 5) after cooldown the failed QXFP5 heater was found to be shorted to the coil and was disconnected from the heater firing unit and from ground. Unfortunately, the heater-to-coil short in coil QXFP5 was a double short, as demonstrated by post-test data analysis [9], [10], and the current going through the heater in the subsequent 17 quenches generated sufficient heat to damage the ground insulation.

At present, the HL-LHC Inner Triplet Electrical Design Criteria [11] clearly state that Hipot tests after coils have been exposed to helium must be performed at a reduced value $(460 \mathrm{~V})$ since helium may be trapped in the coils. When the MQXFAP1 test started, the electrical design criteria had not yet been finalized and the test team followed the LARP procedure of performing high voltage tests at the same values before and after cold test.

It should be noted that only one MQXFAP1 heater developed a short to coil during the $2.5 \mathrm{kV}$ Hipot after exposure to helium. This fact shows that the non-conforming epoxy impregnation of coil QXFP5 was the main cause and the Hipot a secondary cause.

A few other electrical issues were found during fabrication of MQXFA prototype coils and of the very first pre-series coil (QXFA108). The second pre-series coil (QXFA109) developed an electrical issue during magnet assembly and was put on hold for repair. These issues had two separate causes.

A few prototype coils failed the coil-saddle Hipot at $1 \mathrm{kV}$ that is performed after coil fabrication is complete. Coil QXFA109 showed some anomalies when an impulse test was performed at $2.5 \mathrm{kV}$ after coil-pad subassembly. Subsequent electrical tests allowed locating the electrical weakness (between the coil and the saddle in the return-end inner-layer) and further degraded the dielectric strength between these components. The saddles (also known as "end shoes") are the structural parts in contact with coil outermost turn at each end (Fig 1). They are

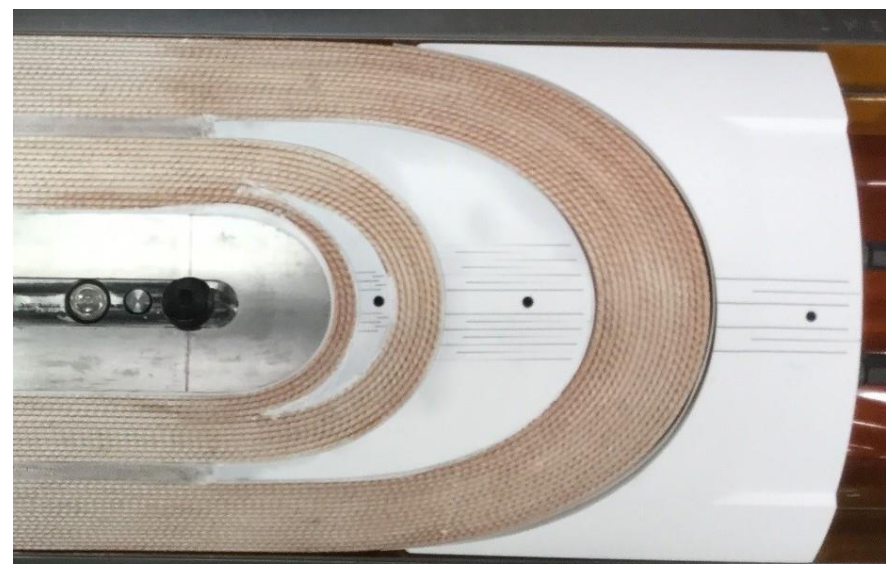

Fig. 1. Photo of an MQXF return end after coil winding and curing. The white component on the right is the saddle, and the other two white parts are end spacers. These parts are white because they are plasma coated using $\mathrm{Al}_{2} \mathrm{O}_{3}$ powder to improve electrical insulation. They have slits for allowing some flexibility during winding. On the left there is the tip of the coil pole made of Ti-6Al$4 \mathrm{~V}$.

made of 316L stainless steel in order to transfer the very large axial forces $(0.3 \mathrm{MN}$ per coil) to magnet structure and are plasma coated using $\mathrm{Al}_{2} \mathrm{O}_{3}$ powder with $200 \mu \mathrm{m} \pm 50 \mu \mathrm{m}$ thickness to increase the dielectric strength. In the present design MQXFA coil saddles have slits (Fig 1), which allow for some flexibility (i.e. saddle tips can get further away or closer) when saddles are installed at the end of winding and during subsequent fabrication steps until the coil is epoxy impregnated. During MQXFA prototype coil fabrication temporary saddles with slits were used during coil curing and they were replaced by permanent saddles without slits before coil heat treatment. This change was done because of concerns about the behavior of saddles with slits under the very large axial forces during magnet operation. This concern was addressed by the successful test of short models [6], [12] using CERN coils with slit saddles. This successful demonstration allowed AUP to make a design change and keep the saddles with slits through all coil fabrication. After this design change no other coil-saddle issue occurred. It demonstrates that these issues were due to cable insulation damage caused by the saddle tips when the solid saddles were inserted after coil curing, together with microcracks of the plasma coating.

A few prototype and pre-series coils failed the coil-pole Hipot that is performed after coil fabrication is complete. This test was performed at $500 \mathrm{~V}$ up to coil QXFA112, and it was reduced to $100 \mathrm{~V}$ for the following coils. The pole is electrically floating and the $100 \mathrm{~V}$ Hipot ensures that the power dissipated through the insulation is less than $1 \mathrm{~mW}$ if both inner-layer and outer-layer pole turns have a short-to-pole.

Coil QXFA108 was the last coil that did not pass this Hipot. It was rejected and used for several tests. Autopsy of coil QXFA108 together with analysis of travelers and pictures, suggested that the cause of this issue was local excessive amount of the ceramic binder (CTD-1202) that is applied on each coil layer before curing [2]. During coil heat treatment, pyrolysis of the binder leaves a slight carbon or other conductive residue [13]. An excess amount of binder in this location led to a 
substantially higher concentration of residue, above the percolation threshold, leading to insufficient insulation strength. This issue has been addressed by reducing the amount of ceramic binder to 1.4 gram per meter of cable and by avoiding the three turns closest to the pole. These changes should also reduce the mechanical strength degradation observed on the S2-glass fibers in contact with the ceramic binder during coil heat treatment [14]. The MQXFB team is experimenting a further reduction of ceramic binder per coil by using it only on coil inner layer [15].

\section{A. First lesson learned}

The fabrication technology in use for MQXF coils was developed by LARP around the 2004-2014 decade by using elements of FNAL [16] and LBNL [17] R\&D programs. This development culminated with the successful LQ [18] and HQ [19] programs. During this time LARP performed Hipot tests to ground up to $1 \mathrm{kV}$, which was the max allowable voltage at the vertical test facilities used by LARP at that time. Moreover, coil-pole and coil-saddle Hipots were introduced only at the end of the LARP program because of the low voltages developed in LARP magnets, which were shorter and with fewer turns per coil than MQXF magnets. Therefore, whereas the LARP electrical QC plan was sufficient for the magnets developed by that program, it did not allow finding some electrical weaknesses subsequently unveiled in MQXF coils and magnets.

The main lesson learned through these issues can thus be summarized: as early as possible during the $R \& D$ phase for magnets to be used in future particle accelerators, try to estimate the peak voltages that these magnets will experience during operation, add sufficient margin to all high-voltage tests (Hipot and Impulse tests), and perform electrical QC tests at these levels.

This plan is clearly challenging because machine layout and quench protection parameters may not be available in the early phases of magnet R\&D. Nonetheless the authors think that the effort to make reasonable guesses will be paid off during subsequent phases of magnet development.

\section{LESSON LEARNED ABOUT DESIGN CRITERIA}

The second prototype (MQXFAP2) demonstrated poor training and limited performance because of a fractured aluminum shell. MQXFAP2 test data were presented in [7], [20] and a detailed analysis of fracture causes and preventive actions was published in [21]. Here we present a short list of the causes, which lead to this issue: 1) the design of the MQXFAP2 aluminum shells included small rounding ( $3 \mathrm{~mm}$ radius) at cutout corners, 2) a vendor made an error during fabrication resulting in "sharp corners" on one side of an end shell, 3) this non-conformity was accepted based on past LARP experience, 4) a small design change, successfully used on some short models, increased the end shell pre-load during cooldown.

When this issue occurred the MQXFA Structural Design Criteria [22] were still under development. These criteria require the use of 10- or $15-\mathrm{mm}$ radii at shell cutouts, ultrasound test after forging, die-penetrant test after machining, and a detailed analysis of non-conformities. The structures used in MQXFA pre-series and series magnets are conforming to these criteria.

\section{A. Second Lesson Learned}

The successful use in many LARP magnets of aluminumshell-based structures may have contributed to excessive confidence when the magnet assembly team faced the non-conforming MQXFAP2 shell. Nonetheless the main cause of that issue is to be found in the lack at that time of criteria and tools (i.e. Failure Assessment Diagram [21]) for assessing criticality of non-conforming parts. These criteria and tools are now available in the MQXFA Structural Design Criteria [22]. It should also be noted that, in the case of the MQXFAP1 electrical issue, the fact that the HL-LHC IT Electrical Design Criteria [11] were not yet fully available played a significant role.

The main lesson learned from these issues is therefore: finalize Design Criteria (Structural, Electrical, for Superconducting elements, ...) in the early phases of the development of magnets to be used in future particle accelerators.

\section{LESSONS LEARNED ABOUT COIL FABRICATION YIELD}

The AUP project is monitoring fabrication yield of several components. Coil fabrication yield is the ratio of coils accepted over coils fabricated. It is computed including percent complete of coils under fabrication, which passed intermediate QC steps, and assuming 50\% acceptance of coils on hold based on past record. In fact, $\sim 50 \%$ of coils previously on hold had been accepted after repair or dedicated analysis. A coil is put on hold if there is a non-conformity or a process parameter is out of spec (for instance: delamination of midplane insulation after epoxy impregnation, or shocks during shipment higher than in specifications) and may be accepted if repair is successful or analysis demonstrates that the coil is not damaged. A coil is rejected if it does not pass final QC tests, or if there is a critical non-conformity (i.e. a non-conformity affecting form, fit, or functions). AUP assumes coil fabrication to start as soon as insulated cable is accepted. Therefore, if a cable is damaged during winding preparation or following steps, it is counted as a coil failure.

In its baseline (BL) AUP assumed $87.5 \%$ coil fabrication yield. This yield is equivalent to one coil failure every eight coils. This assumption was based on LARP experience. The LARP program made five coils for every new quadrupole (i.e. assuming yield $\geq 80 \%$ ) and often changed coil or magnet design. AUP assumed higher yield than LARP because the same coil design and fabrication process were going to be used on several short-model and prototype coils before starting production.

In January 2020 actual coil yield was $\sim 76 \%$ at both AUP coil fabrication sites: BNL (200 series coils) and FNAL (100 series coils). In October 2020, the time this paper was written, MQXFA coil fabrication was $34 \%$ complete and the yield was $\sim 81 \%$. 
TABLE II

NONCONFORMITY PER COIL FABRICATION STATION

\begin{tabular}{|c|c|c|}
\hline Fabrication station & $\operatorname{BNL}(13.7)^{\mathrm{a}}$ & FNAL $(20)^{a}$ \\
\hline Winding & $1 \mathrm{R}$ & $1 H^{b}-2 R$ \\
\hline Curing & $1 \mathrm{R}$ & \\
\hline $\begin{array}{c}\text { Preparation for heat treatment } \\
\text { Heat treatment }\end{array}$ & & \\
\hline $\begin{array}{l}\text { Preparation for epoxy impregnation } \\
\text { Epoxy impregnation }\end{array}$ & $1 \mathrm{H}$ & $1 \mathrm{H}$ \\
\hline Post impregnation finishing & & $1 \mathrm{H}$ \\
\hline Final electrical tests & & $1 \mathrm{R}$ \\
\hline
\end{tabular}

$\# \mathrm{R} / \# \mathrm{H}$ is the number of coils rejected/on hold at each fabrication station.

aNumber of coils fabricated including \% complete of coils under fabrication, which passed intermediate QC steps.

${ }^{\mathrm{b}}$ Coil QXFA120 was put on hold and subsequently rejected due to separate issues during winding.

'This fabrication station includes NbTi lead splicing.

Several unrelated causes contributed to the lower than assumed yield. As shown in Table II, only one fabrication station (coil winding at FNAL) had more than one issue causing rejection of coils QXFA118 and QXFA120. In December 2019, the equipment that caused these issues (Selva winder) was put on hold while coil fabrication continued using a back-up system (rotating table), which had been commissioned and successfully demonstrated during the prototype phase. A review, called to assess risks and mitigations for using the FNAL Selva winder, recommended to stop using it and to elevate the rotating table to main winder at FNAL [23]. This recommendation was accepted by AUP and eight coils have been wound on the rotating table with no issue.

Coil QXFA205 was rejected at BNL because the cable was damaged when it dropped from its guide after a stop for unplanned maintenance, and coil QXFA208 was rejected due to its cable being slightly out of place in the layer jump and being subsequently damaged during coil curing. Coil QXFA108 was rejected at FNAL for the electrical issue described in the previous sections.

Two coils presently on hold had issues during preparation for impregnation: in coil QXFA121 a reacted $\mathrm{Nb}_{3} \mathrm{Sn}$ lead was bent during preparation for splicing to the NbTi lead, and in coil QXFA210 two $\mathrm{Nb}_{3} \mathrm{Sn}$ strands were damaged when excessive solder was removed after splicing the $\mathrm{Nb}_{3} \mathrm{Sn}$ lead to the $\mathrm{NbTi}$ lead. Coil QXFA125 was put on hold after $\mathrm{Nb}_{3} \mathrm{Sn}$ was exposed on coil outer surface while filing excessive epoxy on coil midplane. This operation was sometimes needed because the midplane shims of the impregnation fixture were out of tolerance for both straightness and width. After a long search AUP, with CERN help, was able to procure midplane shims which were in tolerance for straightness and closer to meeting the width specification (37.97 - $38.23 \mathrm{~mm}$ width over $4.6 \mathrm{~m}$ length), and this issue should be prevented in future coils. This is an example of the corrective and preventive actions, which were set in place after every issue and shared among the three laboratories fabricating MQXF coils (BNL, FNAL and CERN).
It should be noted that QA and QC are significantly tighter in AUP than in LARP (for instance, the high voltage withstand tests described in previous section). This fact may have contributed to the low coil yield at the start of AUP production. On the other hand, it may result in higher than assumed yield of the AUP magnets, because coils which would have been used by LARP are rejected by AUP. It should also be added that AUP practice of counting as coil failure any issue causing cable rejection after cable was accepted for coil winding, tends to decrease coil yield with respect to other possible ways to compute cable and coil yields.

\section{A. Third and Fourth Lessons Learned}

Nine short coils and 14 prototype coils were fabricated by AUP before starting pre-series coil production. Nonetheless, the $76 \%-81 \%$ yield shows that AUP was still in the first part of the learning curve during pre-series coil fabrication. As shown in the previous section, the main reasons for this low yield in the pre-series phase are: 1) debugging of equipment used for full size coil production, which is different from the equipment used for short coils (i.e. winding stations); 2) human errors partially explained by the need for more technicians to achieve coil production rate significantly higher than during prototyping phase; 3) tighter QA/QC in AUP than in LARP; 4) AUP practice of counting as coil failure any damage to the cable after it was accepted for winding. Coil fabrication yield is expected to reach the target $87.5 \%$ in the second half of series coil production when it will be out of the learning curve.

Therefore, the lesson learned is that equipment changes from short to long coils and the need to increase technician number may negatively affect coil yield in the early phase of $\mathrm{Nb}_{3} \mathrm{Sn}$ coil production even after prototyping phase.

The second lesson learned is more an advice for future projects planning to fabricate hundreds or even thousands of $\mathrm{Nb}_{3} \mathrm{Sn}$ magnets, for instance for FCC-hh [24]. The present fabrication technology for $\mathrm{Nb}_{3} \mathrm{Sn}$ coils to be used in particle accelerator magnets is very labor intensive and requires skilled technicians for several tasks: coil winding, preparation for reaction and heat treatment, splicing to NbTi leads, and more. This is fine for a small-scale production such as HL-LHC, which requires a few dozen magnets. Nonetheless, the authors think that before scaling up production by one or two orders of magnitude an intense effort should be spent to develop a $2^{\text {nd }}$ generation $\mathrm{Nb}_{3} \mathrm{Sn}$ coil fabrication technology for accelerator magnets, aiming at significant reduction of touch labor. Automation may be part of the solution, nonetheless, preparation for this step will require a remarkable effort and extended time. The development of the present technology took about 20 years (from LARP and precursor activities in US laboratories [16],[17] to MQXF finalization by AUP and CERN [25]). It may be prudent to assume that a similar amount of time will be needed for the $2^{\text {nd }}$ generation technology. 


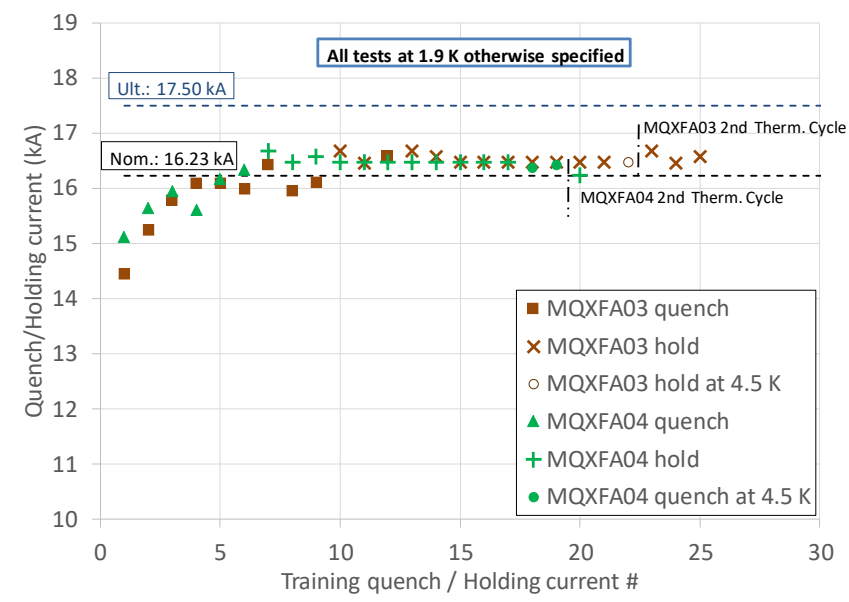

Fig. 2. Training history of first two MQXFA pre-series magnets. Markers labeled "hold" show current that was reached and held for some time without quench.

\section{MQXFA STATUS AND PLANS}

At present AUP has received authorization from the US Department of Energy for conductor procurement, cable fabrication and coil parts procurement. Coil and magnet fabrication have been authorized only partially, and AUP is seeking full approval before spring 2021 in order to maintain schedule.

Strand procurement and cable fabrication are going ahead very well with $68 \%$ strand (RRP $108 / 127$ by B-OST) [26] received, all without significant issues, and $42 \%$ cables accepted with $98 \%$ cable yield (significantly better than $90 \%$ assumed in AUP BL) [27]. From $60 \%$ to $100 \%$ coil parts were received assuring good inventory. Coil fabrication has reached peak production rate both at BNL and at FNAL with one coil completed every 18 days at each laboratory. As previously described, coil yield is $81 \%$ and is lower than the $87.5 \%$ yield assumed in AUP $\mathrm{BL}$, although it is improving. Magnet assembly rate at LBNL is increasing and is expected to reach peak production rate (a magnet completed every 2 months) in the first months of 2021.

The first two pre-series magnets, which are the first MQXFA magnets conforming to MQXFA final design, have been tested at BNL Vertical Test Facility. Both magnets met all key requirements including holding current at nominal current $(16230$ A) plus margin $(300 \mathrm{~A})$. They were also able to reach nominal current after a thermal cycle with no quench. Their training histories (Fig. 2) show numerous current ramps without quench (labeled "hold" in Fig. 2), performed to demonstrate current holding capability and for magnetic measurements, and a few current ramps at $4.5 \mathrm{~K}$ for demonstrating temperature margin. Training was stopped at $16530 \mathrm{~A}$ according to the MQXFA Acceptance Criteria [28]. Two or more upcoming magnets will be trained up to ultimate current (17.5 kA) [28]. Although statistic is still limited, magnet yield is $100 \%$ and is significantly better than the $80 \%$ magnet yield assumed by AUP BL.

AUP is planning to complete assembly of the last MQXFA magnet ( $\# 25$ out of 20 needed for 10 cryo-assemblies) in March 2024.

\section{CONCLUSIONS}

The successful test of the first two pre-series magnets demonstrated AUP readiness for MQXFA series production and is very promising for keeping AUP project within baseline cost and schedule. The lessons learned during prototyping phase and pre-series fabrication were instrumental for this good start. In this paper we shared the most important lessons learned up to present time, focusing on those which may be most useful for other $\mathrm{Nb}_{3} \mathrm{Sn}$ accelerator magnet projects. We think that this sharing is very useful for the entire community and we thank the projects and colleagues who are doing it, in particular the $11 \mathrm{~T}$ team at CERN [29] with whom the MQXF teams are sharing non-conformities, corrective and preventive actions.

\section{ACKNOWLEDGMENT}

The authors would like to thank the many colleagues who contributed to this effort by reviewing the MQXF design and the AUP project. In particular, the authors are very thankful to the AUP CD-1 review committee that recommended the development of Structural Design Criteria for MQXFA magnets.

\section{REFERENCES}

[1] O.Bruning, et al. 2002 LHC Project Report 626

[2] G. Ambrosio, " $\mathrm{Nb}_{3} \mathrm{Sn}$ High Field Magnets for the High Luminosity LHC Upgrade Project", IEEE Trans. Appl. Supercond., vol. 25, 2015.

[3] G. Apollinari, et al., "US Contributions to the High Luminosity LHC Upgrade - Focusing Quadrupoles and Crab Cavities", in Proc. 10th Int. Particle Accelerator Conf. (IPAC'19), Melbourne, Australia, May 2019, pp. 536-539.

[4] S. Izquierdo Bermudez, et al., "Progress in the Development of the $\mathrm{Nb}_{3} \mathrm{Sn}$ MQXFB Quadrupole for the HiLumi Upgrade of the LHC", IEEE Trans. Appl. Supercond., submitted for publication.

[5] S. A. Gourlay, et al., "Magnet R\&D for the US LHC Accelerator Research Program (LARP)," IEEE Transactions on Applied Superconductivity, vol. 16, no. 2, pp. 324-327, June 2006.

[6] S. Stoynev, et al., "Summary of Test Results of MQXFS1-The First Short Model $150 \mathrm{~mm}$ Aperture $\mathrm{Nb}_{3} \mathrm{Sn}$ Quadrupole for the HighLuminosity LHC Upgrade', IEEE Trans. Appl. Supercond., Vol. 28, No 3, 2018, Art no. 4001705

[7] V. Marinozzi, et al. "Study of the heater-coil electrical insulation for the HL-LHC low beta quadrupoles", IEEE Trans. Appl. Supercond., submitted for publication.

[8] J. Muratore et al., "Test Results of the First Two Full-Length Prototype Quadrupole Magnets for the LHC Hi-Lumi Upgrade”, IEEE Trans. Appl. Supercond., Vol. 30, No 4, March 2020, Art no. 4004205.

[9] E. Ravaioli, "Analysis of the short circuit in the MQXFAP1 magnet", CERN-EDMS-2037314, 2018

[10] V. Marinozzi, "Analysis of MQXFAP1 Short-to-ground", Us-HilumiDoc-897, 2018.

[11] F. Menedez Camara, "Electrical design criteria for the HL-LHC inner triplet magnets", CERN-EDMS-1963398, 2018.

[12] P. Ferracin, et al., "The HL-LHC Low- $\beta$ Quadrupole Magnet MQXF: From Short Models to Long Prototypes", IEEE Trans. Appl. Supercond., Vol. 29, No 5, 2019, Art. no. 4001309.

[13] E. Holik, private communication.

[14] M. Gonçalves Lopes, et al., "Glass Fiber/Binder Characterization" CERN TE-MSC Internal Note 2014-18, CERN-EDMS-1405346, 2014.

[15] S. Izquierdo Bermudez and A. Milanese, private communication.

[16] A. V. Zlobin, et al, "R\&D of $\mathrm{Nb}_{3} \mathrm{Sn}$ accelerator magnets at Fermilab", IEEE Trans. Appl. Supercond., Vol. 15, No 2, June 2005, pp. 1113-1118.

[17] G. Sabbi, et al. "Design of racetrack coils for high-field dipole magnets", IEEE Trans. Appl. Supercond., Vol. 11, No 1, March 2001, pp 2280-2283.

[18] G. Ambrosio, et al., "Test Results and Analysis of LQS03 Third Long $\mathrm{Nb}_{3}$ Sn Quadrupole by LARP”, IEEE Trans. Appl. Supercond., Vol. 23, No 3, June 2013. 
[19] J. DiMarco, et al., "Test Results of the LARP $\mathrm{Nb}_{3} \mathrm{Sn}$ Quadrupole HQ03a,", IEEE Trans. Appl. Supercond., Vol. 26, No 4, June 2016.

[20] D. Cheng, et al., "Mechanical Performance of the First Two Prototype 4.5 $m$ Long Nb3Sn Low- $\beta$ Quadrupole Magnets for the Hi-Lumi LHC Upgrade", IEEE Transactions on Applied Superconductivity, vol. 30, no. 4, June 2020, Art no. 4000906.

[21] H. Pan, et al., "Fracture Failure Analysis for MQXF Magnet Aluminum Shells", IEEE Transactions on Applied Superconductivity, vol. 30, no. 4, June 2020, Art no. 4002307.

[22] E. Anderssen and S. Prestemon, "MQXFA Structural Design Criteria", US-HiLumi-doc-909.

[23] V. Lombardo, J. Blowers, L. Elementi "Review of SELVA Winder Risk Assessment and Lessons Learned", US-HiLumi-doc-2958.

[24] D. Tommasini, et al., "Status of the $16 \mathrm{~T}$ dipole development program for a Future Hadron Collider", IEEE Trans. Appl. Supercond., vol. 28, no. 3, Apr. 2018, Art no. 4001305.

[25] P. Ferracin, et al., "Development of MQXF: The $\mathrm{Nb}_{3}$ Sn Low- $\beta$ Quadrupole for the HiLumi LHC," IEEE Trans. Appl. Supercond., vol. 26 no. 4, June 2016, Art. no. 4000207

[26] J. Levitan, et al., "Verification Testing of MQXFA Nb${ }_{3} \mathrm{Sn}$ Wires Procured Under LARP", IEEE Trans. Appl. Supercond., vol. 29 no. 5, Aug 2019, Art. no. 6000904.

[27] I. Pong, et al., "Bare Cable Fabrication Statistics for the AUP MQXF Magnet Coils at 50\% Milestone", IEEE Trans. Appl. Supercond., submitted for publication.

[28] R. Carcagno and E. Todesco, "US HL-LHC AUP Acceptance Criteria PART A: MQXFA Magnet" CERN-EDMS-2031083 v.1.5, 2020

[29] F. Savary, et al. "Design and Construction of the Full-Length Prototype of the 11-T Dipole Magnet for the High Luminosity LHC Project at CERN”, IEEE Trans. Appl. Supercond., vol. 28, no. 3, Apr. 2018, Art no. 4007106 